\title{
Kv1.3 channel blockade enhances the phagocytic function of RAW264.7 macrophages
}

\author{
ZHU Hong ${ }^{1},{\text { YAN } \mathrm{Li}^{2}, \text { GU JingLi }}^{1}, \mathrm{HAO} \mathrm{Wei}^{1} \&$ CAO JiMin ${ }^{1 *}$ \\ ${ }^{1}$ Department of Physiology, Institute of Basic Medical Sciences, Chinese Academy of Medical Sciences; School of Basic Medicine Peking, \\ Union Medical College, Beijing 100005, China; \\ ${ }^{2}$ Department of Pathophysiology, Institute of Basic Medical Sciences, Chinese Academy of Medical Sciences; School of Basic Medicine, \\ Peking Union Medical College, Beijing 100005, China
}

Received April 30, 2015; accepted June 13, 2015

\begin{abstract}
This study aimed to comprehend the largely unknown role of voltage-gated potassium channel 1.3 (Kv1.3) in the phagocytic function of macrophages. We found that blocking of the Kv1.3 channel with $100 \mathrm{pmol} \mathrm{L}^{-1}$ Stichodactyla helianthus neurotoxin (ShK) enhanced the phagocytic capacities of both resting and lipopolysaccharide (LPS)-stimulated RAW264.7 macrophages in the chicken erythrocyte system. In the fluorescein isothiocyanate (FITC)-labeled Escherichia coli k-12 system, ShK increased the phagocytic capacities of resting RAW264.7 cells, but not of the LPS-stimulated cells, as LPS alone stimulated almost saturated phagocytosis of the macrophages. ShK increased the nitric oxide (NO) production in LPS-activated cells, but not in resting RAW264.7 cells. There was no effect of ShK alone on the cytokine secretions in resting RAW264.7 cells, but it suppressed IL-1 $\beta$ secretion in LPS-stimulated RAW264.7 cells. At a concentration of 100 pmol L ${ }^{-1}$, ShK did not affect the viability of the tested cells. Kv1.3 was expressed in RAW264.7 cells; this expression was downregulated by LPS, but significantly upregulated by disrupting caveolin-dependent endocytosis with filipin III. In addition, cytochalasin D, an inhibitor of actin polymerization, did not affect the Kv1.3 expression. Thus, blocking of the Kv1.3 channel enhances the phagocytic capacity and NO production of this cell line. Our results suggest that Kv1.3 channel serves as a negative regulator of phagocytosis in macrophages and can therefore be a potential target in the treatment of macrophage dysfunction.
\end{abstract}

\section{Kv1.3 channel, phagocytosis, macrophage activation syndrome, immunoregulation}

Citation: Zhu H, Yan L, Gu JL, Hao W, Cao JM. Kv1.3 channel blockade enhances the phagocytic function of RAW264.7 macrophages. Sci China Life Sci, 2015, 58: 867-875, doi: 10.1007/s11427-015-4915-3

Ion channels are expressed in both excitable cells and non-excitable cells. Compared with those in the excitable cells, the functions of ion channels in the non-excitable cells, such as immune cells, are not well documented. In immune cells, ion channels play key roles in the signal transduction chains of their immune activities. Several types of ion channels have been reported in $\mathrm{T}$ lymphocytes, including voltage-gated $\mathrm{K}^{+}$channel 1.3 (Kv1.3), $\mathrm{Ca}^{2+}$ activated $\mathrm{K}^{+}$channel $3.1(\mathrm{KCa} 3.1), \mathrm{Ca}^{2+}$ release-activated $\mathrm{Ca}^{2+}$ (CRAC) channel, transient receptor potential me-

*Corresponding author (email: caojimin@126.com) lastatin 7 (TRPM7) channel, and $\mathrm{Cl}^{-}$channel [1]. Kv channels are widespread in various immune cells, including macrophages, and their actions can avoid membrane over depolarization and maintain the normal cell functions [2].

Macrophage plays an important role in the immune activities via phagocytosis, antigen-presentation, cytokines release, regulation of $\mathrm{T}$ cell signaling [3] and clearance of apoptotic neutrophils [4], and is involved in the pathogenesis of many immune- or autoimmune-related diseases including atherosclerosis, vasculitis and hypertension $[5,6]$. Similar to that in excitable cells, changes in the membrane potential are among the earliest events that occur in re- 
sponse to stimulation [7] and thereby affect the functional activation state of macrophages. Various ion channels has been reported in macrophages, including Kv1.3 [8], Kv1.5 [8], inwardly rectifying $\mathrm{K}^{+}$channel 2.1 (Kir2.1) [7], KCa3.1 [9], CRAC [10], transient receptor potential cation (TRPC) channel [11], and voltage-gated proton (hydrogen) channels (HVCN) [12]. Among these channels, the Kv channels play a pivotal role in the modulation of macrophage physiology [13]. For example, certain voltage-dependent $\mathrm{K}^{+}$channels, such as $\mathrm{Kv1.3,} \mathrm{Kv1.5,} \mathrm{Kir2.1,} \mathrm{and} \mathrm{outward} \mathrm{delayed} \mathrm{rectifier} \mathrm{K}^{+}$ channel, demonstrate differential responses during macrophage proliferation and activation [7]; the expression pattern of $\operatorname{Kv} \beta$ subunit is dependent on the proliferation and activation mode in macrophages [14]; Kv1.3 and Kv1.5 co-associate and generate functional heterotetramers in macrophages [8]; selective Kv1.3 blockade affects the expression levels of some important cholesterol-metabolismassociated molecules (scavenger receptor class A, lectinlike oxidized low-density lipoprotein receptor-1, acetylcoenzyme A acetyltransferase 1 (ACAT1), and ATPbinding cassette transporter A1 (ABCA1)) in human macrophages exposed to ox-LDL [15]; Kv1.3 and Kv1.5 are involved in the induction of macrophage apoptosis [16]; and Kv1.3 modulates macrophage migration [17]. Kv1.3 channel is also involved in the maintenance of membrane potential, $\mathrm{Ca}^{2+}$ signaling, proliferation, and activation of immune cells such as macrophages $[18,19]$. Furthermore, it has been suggested that Kv1.3 may serve as a novel target in therapies of asthma [20] and atherosclerosis [21].

Despite the knowledge about these facts, the role of Kv1.3 in the phagocytic function of macrophage has rarely been investigated. In this study, we focused on comprehending the phagocytic function of macrophages using RAW264.7 cell line as the macrophage model to observe the role of Kv1.3 in the phagocytosis of macrophages on chicken erythrocytes and Escherichia coli k-12. As selective Kv1.3 channel agonist is not commercially available, we used Stichodactyla helianthus neurotoxin (ShK), a selective Kv1.3 channel blocker [22], to manipulate the function of Kv1.3 channels. Our key results demonstrated that selective blockade of Kv1.3 channel could increase the phagocytic capacity of RAW264.7 cells, which suggests that Kv1.3 may serve as a suppresser or negative regulator of macrophage phagocytosis. This novel finding can significantly contribute toward the development of therapeutic strategies for diseases involving macrophage malfunction.

\section{Materials and methods}

\subsection{Reagents}

Stichodactyla helianthus neurotoxin (ShK) was obtained from Bachem (Swiss); lipopolysaccharide (LPS), cytochalasin D, filipin III, and antibody against Kv1.3 was pur- chased from Sigma (USA); horseradish peroxidaseconjugated secondary antibody from Santa Cruz Biotechnology, Inc. (USA); 4',6-diamidino-2-phenylindole (DAPI), fluo-3/AM, and fluorescein isothiocyanate (FITC)-labeled E. coli k-12 kit from Molecular Probes (USA); TNF- $\alpha$ enzyme linked immunosorbent assay (ELISA) kit from Shanghai ExCell Biology (Shanghai); ELISA assay kits for IL-6 and IL-1 $\beta$ and NO assay kit from Beijing 4A Biotech Co., Ltd. (Beijing); cell counting kit-8 (CCK-8) from Dojindo (Japan); regular reagents for Western blot were obtained from Solarbio SciePVDFe \& Technology Inc., Ltd., (Beijing); protein marker from New England Biolabs (Beijing); BCA protein assay kit and antifluorescence quenching agents from Beyotime Biotechnology Inc. Ltd., (Shanghai); and Switzerland and Giemsa staining solutions from Beijing Solarbio Science \& Technology Co., Ltd. (Beijing).

\subsection{Cell culture}

The RAW264.7 cell line was purchased from the Cell Center, Chinese Academy of Medical Sciences. Post-recovery, the RAW264.7 cells were cultured in Dulbecco's modified Eagles medium (DMEM) supplemented with $10 \%$ fetal bovine serum, $100 \mathrm{U} \mathrm{mL}^{-1}$ penicillin, and $100 \mathrm{mg} \mathrm{mL}^{-1}$ streptomycin. The culture was maintained at $37^{\circ} \mathrm{C}$ under a $5 \%$ $\mathrm{CO}_{2}$ humidified atmosphere. Cells at $3 \times 10^{3} \mathrm{~mL}^{-1}$ concentration in DMEM medium were plated onto 96-well plates and allowed to adhere overnight. The cells were passaged and cultured to achieve $80 \%$ confluence for further study.

\subsection{Preparation of chicken erythrocytes}

We used nucleated chicken erythrocytes to study the role of Kv1.3 in the phagocytic activities of RAW264.7 macrophages. Chicken blood sample was harvested at a chicken farm. To isolate the erythrocytes, $5 \mathrm{~mL}$ of blood was draw from the chicken artery with a syringe and then transferred to heparinized tubes prefilled with $20 \mathrm{~mL}$ of Alsever solution (containing anticoagulant sodium citrate and glucose) to obtain a blood:Alsever solution ratio of 1:4, followed by mixing and storage at $4^{\circ} \mathrm{C}$. The blood samples were washed with sterile saline thrice and then centrifuged. The supernatant and white blood cells were discarded, and the remaining erythrocytes were added to sterile saline, with a final volume to $4 \mathrm{~mL}$. The erythrocyte solution was recentrifuged, and then one drop of erythrocytes was added to sterile saline to make $1 \%$ concentration of the cell suspension.

\subsection{Cell viability assay}

The viability of the RAW264.7 cells was determined by the CCK-8 assay. To examine the role of Kv1.3 in phagocytosis, the RAW264.7 cells were pre-incubated with ShK at 
different concentrations for $24 \mathrm{~h}$ in a 96-well plate. Cells treated with saline served as control. After incubation, the RAW264.7 cells were cultured for $2 \mathrm{~h}$ in $10 \mu \mathrm{L}$ CCK-8containing culture medium. Next, the absorbance was measured at $450 \mathrm{~nm}$ with a reference wavelength of $630 \mathrm{~nm}$ on a microplate reader (Synergy 4, BioTek, USA). The cell viability was expressed as the percentage of control, which was defined to be $100 \%$.

\subsection{Phagocytosis assay and Switzerland Giemsa stain- ing of chicken erythrocytes}

Mature chicken erythrocytes possess nuclei that can be easily recognized under a microscope (Nikon E100, Japan) when phagocytosed. Switzerland Giemsa staining of chicken erythrocytes is an established method that allows explicit and quantitative measurement of cell phagocytosis. To observe the phagocytosis of RAW264.7 cells on chicken erythrocytes, the RAW264.7 cells (with or without ShK pretreatment) and chicken erythrocytes were co-cultured for $2 \mathrm{~h}$ and then washed with phosphate-buffered solution (PBS) to remove the erythrocytes not adhering to the RAW264.7 cells.

For Giemsa staining, the RAW264.7 cells were treated with Switzerland staining solution for $2 \mathrm{~min}$ at room temperature. After the staining solution turned red, an equivalent amount of PBS was added and the staining process was continued for an additional $10 \mathrm{~min}$. The cells were then washed with PBS, air-dried, and stained with Giemsa solution for $4 \mathrm{~min}$ at the room temperature. The cells were then washed with distilled water thrice, air-dried, and covered with neutral gum. The phagocytosed chicken erythrocytes presenting inside the RAW264.7 cells were examined under a microscope by a person blinded to the experimental design. Three fields of each coverslip were randomly selected, and all phagocytosed chicken erythrocytes on each field were counted. The phagocytosis rate (PR), which reflects the mean numbers of phagocytosed erythrocytes in each RAW264.7 cell, was calculated according to the following equation: $\mathrm{PR}=$ (total numbers of the phagocytosed erythrocytes in 200 RAW264.7 cells) $/ 200 \times 100 \%$.

\subsection{Flow cytometry to quantitate the phagocytosis of RAW264.7 cells on FITC-labeled $E$. coli $\mathrm{k}-12$}

An alternative measure was used to examine the phagocytosis of the RAW264.7 cells on E. coli k-12 labeled with FITC. The fluorescence intensity inside the RAW264.7 cells served as an indicator of phagocytic capacity. Briefly, cells culture and treatment procedures were kept the same as described above, except that the chicken erythrocytes were replaced by FITC-labeled $E$. coli k-12. After co-culturing the RAW264.7 cells with FITC- labeled E. coli k-12 for different time periods followed by washing with
PBS, the RAW264.7 cells were loaded into the FACSCallibur flow cytometer (Becton Dickinson, USA) and the fluorescence of FITC-labeled E. coli inside the RAW264.7 cells was measured with the absorption/emission maxima of $560 / 585 \mathrm{~nm}$ at the FL-1 channel. The mean fluorescence intensity (MFI) was calculated by using the ImageJ software, and the data for fluorescence activating cell sorter (FACS) were analyzed by the software FCS-Express version 3.0 (De Novo).

Two parameters of phagocytosis were calculated based on the flow cytometry data. (i) The phagocytosis index, which represents the MFI of the RAW264.7 cells, demonstrating phagocytic activities; this parameter was an automatic readout of the flow cytometer and reflected the mean numbers of engulfed FITC-labeled E. coli k-12 in the RAW264.7 cells. (ii) The percentage phagocytosis, which is a parameter reflecting the number of RAW264.7 cells exhibiting phagocytic activities in the entire pool of RAW264.7 cells. In practice, the negative control is considered as the reference region, and a line is drawn at the right-hand side of this region. Cells distributed at the left-hand side of the line were identified as RAW264.7 cells without any phagocytic activities, while cells at the right-hand side of the line were identified as RAW264.7 cells with phagocytic activities.

\subsection{NO assay}

The NO production of the RAW264.7 cells under different treatment conditions was estimated by using a NO assay kit. Briefly, the supernatant $(100 \mu \mathrm{L})$ of $16 \mathrm{~h}$ culture was prepared, and the nitrite content was measured by absorbance at $530 \mathrm{~nm}$ within $30 \mathrm{~min}$ of the last incubation. Nitrite concentration in the samples was calculated with reference to a standard curve prepared with $\mathrm{KNO}_{3}$.

\subsection{ELISA assays of TNF- $\alpha$, IL-6, and IL-1 $\beta$}

At the end of the 16-h stimulation of the RAW264.7 cells with different agents, the culture supernatants were harvested and the levels of TNF- $\alpha$, IL- 6 , and IL- $1 \beta$ in the supernatants was measured by using the respective ELISA assay kits, according to the manufacturer's instructions. Data reflected the secretion levels of these cytokines.

\subsection{Western blot}

The Kv1.3 protein expression levels of the RAW264.7 cells were determined by Western blot. Briefly, the RAW264.7 cells were scraped and collected by centrifugation at $88.8 \times g$ for $5 \mathrm{~min}$ at $4^{\circ} \mathrm{C}$ and resuspended in a radioimmunoprecipitation assay (RIPA) lysis buffer containing $50 \mathrm{mmol} \mathrm{L}^{-1}$ Tris- $\mathrm{HCl}$ (pH 7.4), 150 mmol L ${ }^{-1} \mathrm{NaCl}, 1 \%$ Triton $\mathrm{X}-100$, 
$1 \%$ sodium deoxycholate, $0.1 \%$ sodium dodecyl sulfate (SDS), $2 \mathrm{mmol} \mathrm{L}^{-1}$ ethylenediaminetetraacetate (EDTA), and a series of protease inhibitors including $1 \mathrm{mmol} \mathrm{L}^{-1}$ 4-(2-aminoethyl)benzenesulfonyl fluoride hydrochloride (AEBSF), $800 \mathrm{nmol} \mathrm{L}^{-1}$ aprotinin, $50 \mu \mathrm{mol} \mathrm{L} \mathrm{L}^{-1}$ bestanin, $15 \mu \mathrm{mol} \mathrm{L}{ }^{-1}$ E64, $20 \mu \mathrm{mol} \mathrm{L}{ }^{-1}$ leupeptin and $10 \mu \mathrm{mol} \mathrm{L}^{-1}$ pepstatin A. After thorough lysis on ice, the solution was centrifuged at $12,787 \times g$ for $10 \mathrm{~min}$ at $4^{\circ} \mathrm{C}$ and the supernatant was collected. The protein content was determined by the bicinchoninic acid (BCA) method using samples aliquoted and stored at $-80^{\circ} \mathrm{C}$ until use for $\mathrm{Kv} 1.3$ protein detection. A total of $50 \mu \mathrm{g}$ of the total protein was separated on $10 \%$ SDS-polyacrylamide gel electrophoresis (PAGE) and then transferred onto polyvinylidene fluoride (PVDF) membrane, followed by blocking and incubation with anti-Kv1.3 primary antibody $(1: 1,000)$ at $4^{\circ} \mathrm{C}$ overnight. The blots obtained were incubated with horseradish peroxidase-conjugated secondary antibody $(1: 5,000)$ at the room temperature for $1 \mathrm{~h}$, followed by development on the ECL system (Engreen Biosystem Co, Ltd., Beijing). Images were captured using the EC3 Imaging System (UVP Inc., Upland, USA) and quantified by Quantity One software. The influences of LPS $\left(10 \mu \mathrm{g} \mathrm{mL}^{-1}\right.$ for $\left.10 \mathrm{~h}\right)$, cytochalasin $\mathrm{D}\left(10 \mu \mathrm{gL}^{-1}\right.$ for $1 \mathrm{~h})$, and filipin III $\left(5 \mu \mathrm{g} \mathrm{mL} \mathrm{m}^{-1}\right.$ for $\left.1 \mathrm{~h}\right)$ pre-incubations on the Kv1.3 protein expression of the RAW264.7 cells were measured.

The experimental use of animals in this study was approved by the Life Ethics Committee of Peking Union Medical College, and the protocol followed was in compliance with the US National Institutes of Health Guidelines for the Care and Use of Laboratory Animals (NIH Publication 85-23).

\subsection{Statistical analyses}

Data were presented as $\bar{x} \pm \mathrm{SD}$. Statistical analyses were performed using student's $t$ test or analysis of variance, followed by Newman-Keuls multiple comparisons. $P<0.05$ was considered to be statistically significant.

\section{Results}

\subsection{Blocking of Kv1.3 channel increases phagocytosis of chicken erythrocytes in RAW264.7 cells}

We first adopted the classical chicken erythrocyte method to pharmacologically evaluate the role of Kv1.3 channel in the phagocytosis of RAW264.7 macrophages. For this purpose, we used selective Kv1.3 channel blocker ShK. Our results showed that the control RAW264.7 cells (without ShK and/or LPS treatment) demonstrated weak phagocytic activities, with most of the RAW264.7 cells not showing any phagocytic behaviors on chicken erythrocytes (Figure 1A),

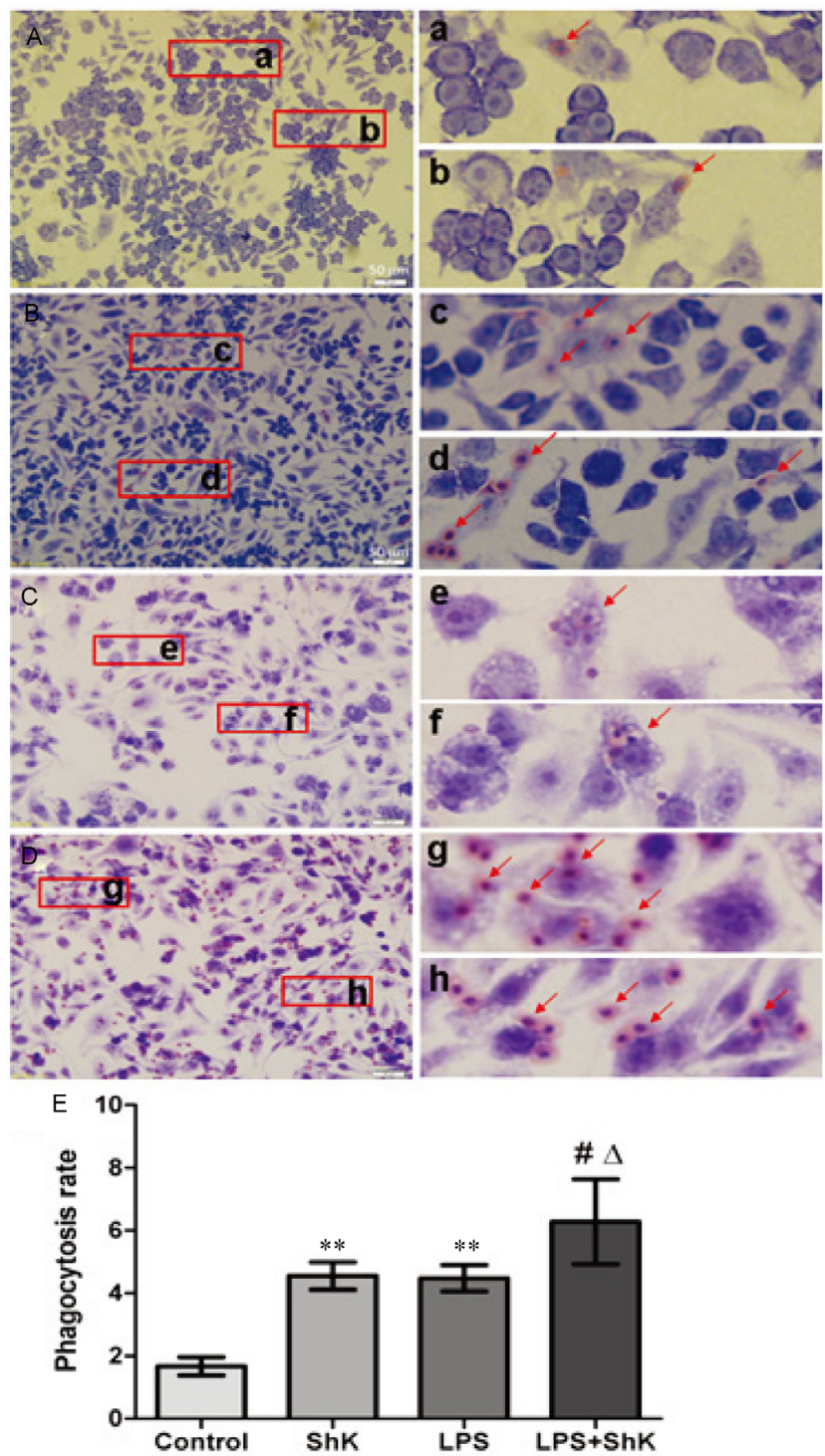

Figure 1 Typical microscopic images of Switzerland Giemsa staining showing the phagocytic activities of RAW264.7 cells on chicken erythrocytes under different conditions. A, Resting (control) untreated RAW264.7 cells showing extremely weak phagocytic activities. B, RAW264.7 cells treated with $\mathrm{ShK}\left(100 \mathrm{pmol} \mathrm{L}^{-1}\right)$ for $2 \mathrm{~h}$ exhibiting enhanced phagocytosis. C, RAW264.7 cells stimulated with LPS $\left(10 \mu \mathrm{g} \mathrm{mL}^{-1}\right)$ for $16 \mathrm{~h}$ also showing enhanced phagocytosis. D, RAW264.7 cells treated with LPS (10 $\mu \mathrm{g}$ $\mathrm{mL}^{-1}$ for $\left.16 \mathrm{~h}\right)$ and $\mathrm{ShK}\left(100 \mathrm{pmol} \mathrm{L}{ }^{-1}\right)$ displaying a further enhancement of phagocytosis in comparison with that of cells treated with LPS or ShK alone. The subpanels marked $\mathrm{a}-\mathrm{h}$ on the right-hand side of the figure are enlarged images corresponding to the framed areas (also marked $\mathrm{a}-\mathrm{h}$ ) on the left-hand side of the figure. The cytoplasm of chicken erythrocytes was stained red, while their nuclei were stained blue (arrows). E, Statistical summary of the PR for different treatment conditions. **: $P<0.01$, vs. Control; \#: $P<0.05$, vs. ShK; $\Delta$ : $P<0.05$, vs. LPS; $n=3$ for each group.

and the PR was found to be only $(1.67 \pm 0.29) \% \quad(n=3)$. However, the RAW264.7 cells pretreated with ShK (100 pmol $\mathrm{L}^{-1}$ for $2 \mathrm{~h}$ ) (the concentration of ShK was consistently $100 \mathrm{pmol} \mathrm{L}^{-1}$ throughout the study) demonstrated an increased phagocytic behavior (Figure 1B), and the numbers 
of phag-ocytosed chicken erythrocytes in single RAW264.7 cells were significantly increased because a single RAW264.7 cell can engulf several chicken erythrocytes at the same time (Figure 1B); the respective PR was increased to $(4.55 \pm 0.44) \%(P<0.01$ vs. control, $n=3)$. LPS $\left(10 \mu \mathrm{g} \mathrm{mL}^{-1}\right)$ (the concentration of LPS was consistently $10 \mu \mathrm{g} \mathrm{mL} \mathrm{m}^{-1}$ throughout the study), a classical stimulator of inflammation and phagocytosis, evidently enhanced the phagocytic activities of the RAW264.7 cells on chicken erythrocytes, as was expected (Figure 1C), and the PR increased to $(4.48 \pm 0.42) \% \quad\left(P<0.01\right.$ vs. control). ShK $\left(100 \mathrm{pmol} \mathrm{L}^{-1}\right)$ further enhanced the phagocytic activities of LPS-stimulated RAW264.7 cells, and the numbers of phagocytosed chicken erythrocytes in single RAW264.7 cells were significantly increased under this condition (Figure 1D) in comparison with those under LPS treatment alone (Figure 1C), with the PR increased to $(6.28 \pm 1.36) \%$ $(P<0.05$ vs. LPS alone). Figure $1 \mathrm{E}$ presents the statistical summary of the PRs under different treatment conditions.

\subsection{Blocking of Kv1.3 channel enhances the phagocy- tosis of $E$. coli $\mathrm{k}-12$ in RAW264.7 cells}

As engulfment and elimination of bacteria is an innate immunological behavior of macrophages, we examined the phagocytic activity of RAW264.7 cells on FITC-labeled $E$. coli $\mathrm{k}-12$ as well as the role of Kv1.3 in this activity by using quantitative flow cytometry assay. Our results demonstrated that selective blockade of Kv1.3 channels by ShK (100 pmol $\mathrm{L}^{-1}$ for $2 \mathrm{~h}$ ) increased the phagocytic capacity of LPS-untreated RAW264.7 cells on FITC-labeled E. coli $\mathrm{k}-12$, as indicated by the increased fluorescence intensity inside the RAW264.7 cells (Figure 2B) as compared with that of the control (Figure 2A and 2E). LPS alone $\left(10 \mu \mathrm{gL} \mathrm{m}^{-1}\right.$ for $16 \mathrm{~h}$ ) could also significantly stimulate the phagocytic activities of RAW264.7 cells on FITC-labeled E. coli k-12 (Figure 2C), and this effect was greater than that of ShK in terms of phagocytosis index (Figure 2E). ShK (100 pmol L ${ }^{-1}$ for $2 \mathrm{~h}$ ) appeared to increase the fluorescence intensity in LPS-pre-stimulated RAW264.7 cells (Figure 2D and 2E), although the difference was not statistically significant $(P>0.05$ vs. LPS alone) (Figure $2 \mathrm{E}$ ).

Alternatively, flow cytometry was used to evaluate the phagocytic capacity of macrophages in order to calculate the percentage of RAW264.7 macrophages displaying phagocytosis (the percentage phagocytosis). As shown in Figure 3, ShK (100 pmol L $\left.{ }^{-1}\right)$ significantly increased the percentage phagocytosis. In addition, as a positive control drug, LPS $\left(10 \mu \mathrm{g} \mathrm{mL}^{-1}\right)$ also strongly increased the percentage phagocytosis. No further increase in the percentage phagocytosis was observed when ShK was co-applied with LPS in comparison with the corresponding effect of LPS application alone, probably because a saturation percentage (or a peak) was reached at $>94 \%$ in the LPS-stimulated
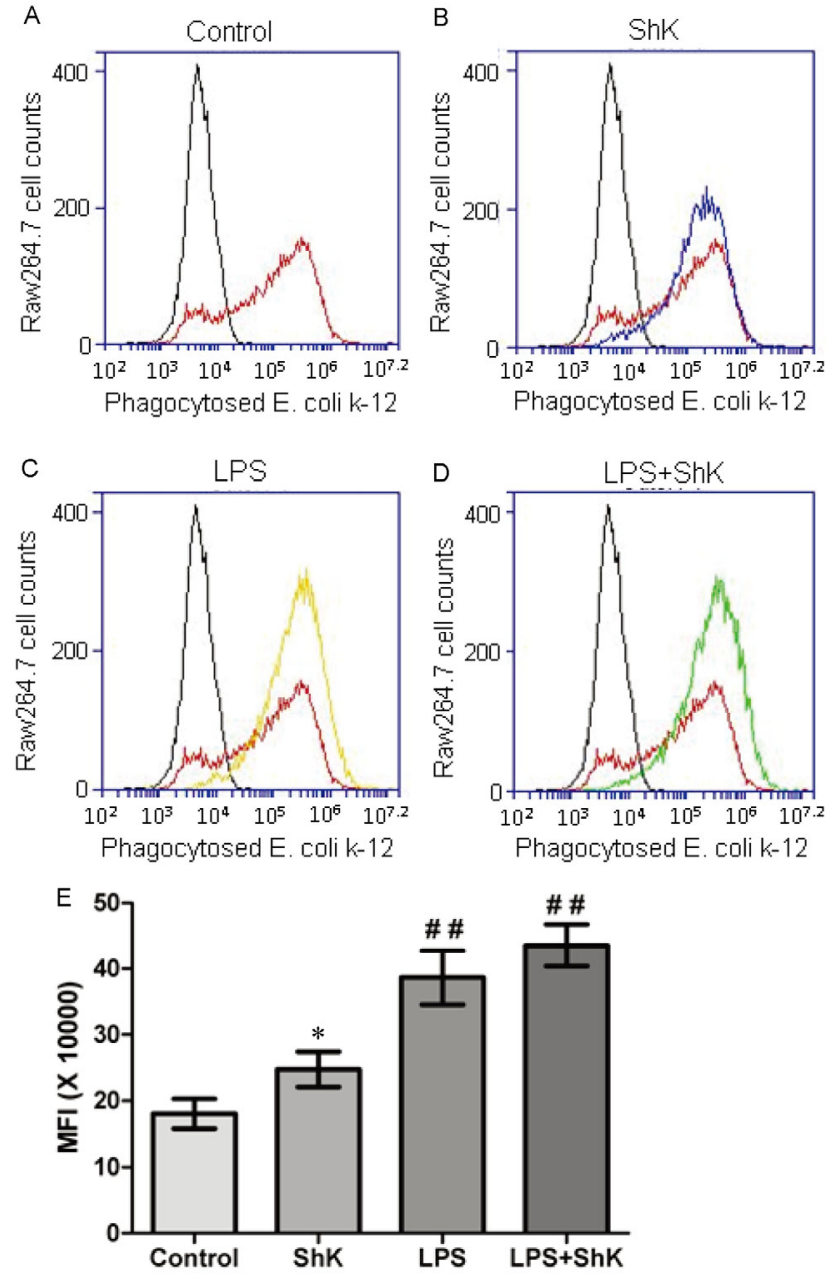

Figure 2 Flow cytometry assay for studying the phagocytic capacities of RAW264.7 cells on FITC-labeled E. coli k-12 under different treatment conditions. The phagocytosis index reflecting the phagocytic capacity was indicated by the MFI. A, Resting (control) RAW264.7 cells showing weak phagocytic activities on the E. coli k-12 system. Black curve and red curve represent the phagocytosis of RAW264.7 cells on FITC-unlabeled and FITC-labeled E. coli k-12, respectively. B, Enhanced phagocytosis of ShK (100 pmol L ${ }^{-1}$ for $2 \mathrm{~h}$ )-pretreated RAW264.7 cells on FITC-labeled E. coli (blue curve) in comparison to that of ShK-untreated cells (red curve). C, LPS (10 $\mu \mathrm{g} \mathrm{mL}^{-1}$ for $16 \mathrm{~h}$ )-stimulated RAW264.7 cells exhibiting enhanced phagocytosis on FITC-labeled E. coli (yellow curve). D, RAW264.7 cells pre-treated with ShK (100 pmol L ${ }^{-1}$ for $\left.2 \mathrm{~h}\right)$ and LPS (10 $\mu \mathrm{g} \mathrm{mL} \mathrm{m}^{-1}$ for $16 \mathrm{~h}$ ) yielding similar enhancement in phagocytosis as that with LPS treatment alone $(P>0.05)$. E, Statistical bar graph reflecting the MFI in each treatment group $(n=3)$, indicating the quantities of engulfed $E$. coli. *: $P<0.05$, vs. Control; \#\#: $P<0.01$ vs. ShK.

RAW264.7 cells. These results are consistent with those of the erythrocyte assay, suggesting that Kv1.3 may be a negative regulator of the phagocytosis of RAW264.7 cells.

\subsection{Blocking of Kv1.3 channel increases NO produc- tion in LPS-stimulated RAW264.7 cells}

As nitric oxide (NO) is an important indicator of macrophage activation, we studied the NO production by 

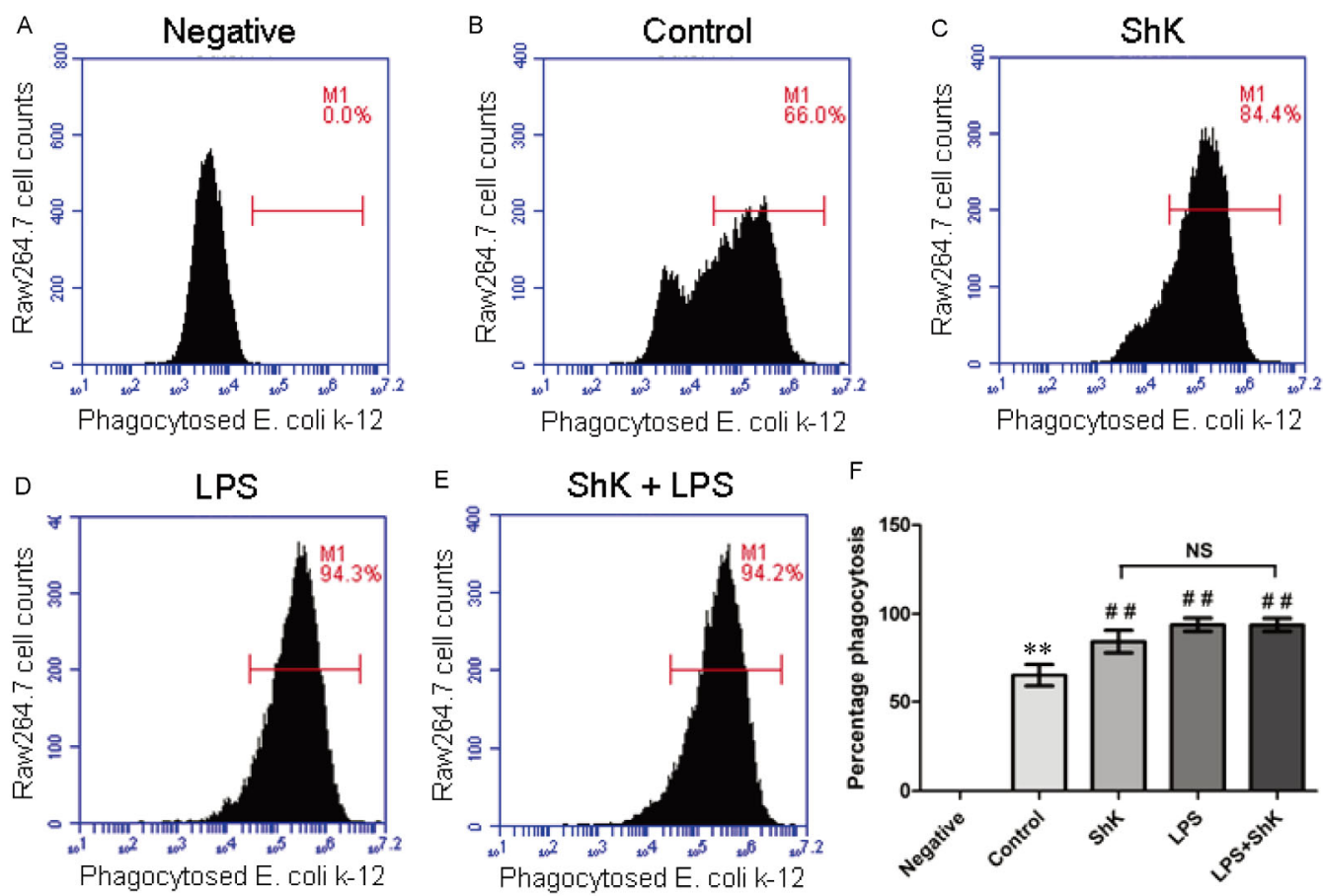

Figure 3 Flow cytometry assay was performed to evaluate the percentage phagocytosis of RAW264.7 cells under different treatment conditions. A-E, Represents the results of flow cytometry. Negative, the E. coli k-12 system was not labeled with FITC, therefore the phagocytosis percentage was set to $0.0 \%$. Control, RAW264.7 cells without drug treatment, but with E. coli $\mathrm{k}-12$ labeled with FITC, indicating that the resting cells demonstrated phagocytic behaviors to some extent. ShK, RAW264.7 cells treated with ShK (100 pmol L $\left.{ }^{-1}\right)$. LPS, RAW264.7 cells treated with LPS (10 $\mu \mathrm{g}$ mL $\left.{ }^{-1}\right)$. ShK+LPS, RAW264.7

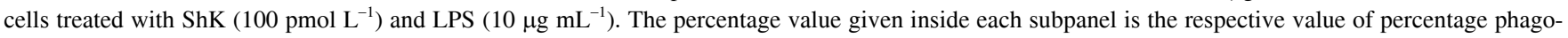
cytosis. The red line segment in each subpanel indicates the area in which the phagocytosed FITC-labeled E. coli k-12 would appear. F, Statistical summary of the percentage phagocytosis for different treatments ( $n=3$ for each treatment). $* *: P<0.01$, vs. Negative; \#\#: $P<0.01$, vs. Control; NS: not significant.

RAW264.7 cells under different treatment conditions. Compared with the control (resting) cells, LPS $\left(10 \mu \mathrm{g} \mathrm{mL}{ }^{-1}\right.$ for $16 \mathrm{~h}$ )-stimulated RAW264.7 cells showed significant increase in the NO production, with an increase in the NO level from $(1.85 \pm 0.01) \mathrm{mmol} \mathrm{L}^{-1}$ (control) to $(35.11 \pm 0.87$ ) mmol L ${ }^{-1}(P<0.01)$ (Figure 4A), suggesting that, LPS, a known activator of macrophages, can significantly stimulate NO production in RAW264.7 cells. ShK (100 pmol L $\left.{ }^{-1}\right)$ alone could not stimulate the NO production in resting RAW264.7 cells (Figure 4A), but it enhanced the NO production in LPS-stimulated RAW264.7 cells (Figure 4A), with an increase from the control level of $(35.11 \pm 0.87) \mathrm{mmol} \mathrm{L}^{-1}$ to $(41.27 \pm 0.42) \mathrm{mmol} \mathrm{L}^{-1}(P<0.05)$ (Figure $\left.4 \mathrm{~A}\right)$. These results are consistent with those of the phagocytosis assays, together suggesting that activation of Kv1.3 channel may exert an inhibitory effect on NO production of RAW264.7 cells upon LPS stimulation.

2.4 Blocking of Kv1.3 channel did not significantly affect cell viability and secretions of TNF- $\alpha$ and IL-6, but it decreased LPS-induced IL-1 $\beta$ secretion in RAW264.7 cells

CCK-8 assay was performed to assess the role of Kv1.3 in macrophage viability. ShK treatment (100 pmol L ${ }^{-1}$ for $\left.24 \mathrm{~h}\right)$ did not significantly affect the viability of RAW264.7 cells (Figure 4B).

Double antibodies-sandwich enzyme-linked immunosorbent assay (ELISA) was performed on the culture supernatant to examine cytokine secretion. As TNF- $\alpha$ is an inflammatory cytokine released by macrophages on activation, we also accessed whether Kv1.3 plays a role in TNF- $\alpha$ production by RAW264.7 cells. ShK (100 pmol L $\left.{ }^{-1}\right)$ alone could not induce TNF- $\alpha$ release relative to the control (Figure $4 \mathrm{C}$ ). As a well-known stimulator of TNF- $\alpha$ released in macrophages, LPS $\left(10 \mathrm{pg} \mathrm{mL}^{-1}\right)$ significantly increased the secretion of TNF- $\alpha$ by RAW264.7 cells than that of LPS-untreated control cells (Figure 4C). At the same concentration, ShK treatment could not further increase the TNF- $\alpha$ production in LPS-stimulated RAW264.7 cells (Figure 4C), probably because LPS had already stimulated maximal TNF- $\alpha$ production or that ShK did not have any effect on the TNF- $\alpha$ production.

LPS $\left(10 \mu \mathrm{g} \mathrm{mL}^{-1}\right)$ significantly increased the secretions of IL-6 (Figure 4D) and IL-1 $\beta$ (Figure 4E) in RAW264.7 cells. ShK (100 pmol L $\left.{ }^{-1}\right)$ alone did not significantly affect the secretions of IL-6 (Figure 4D) and IL-1 $\beta$ (Figure 4E), rather suppressed the LPS-induced secretion of IL-1 $\beta$ in 

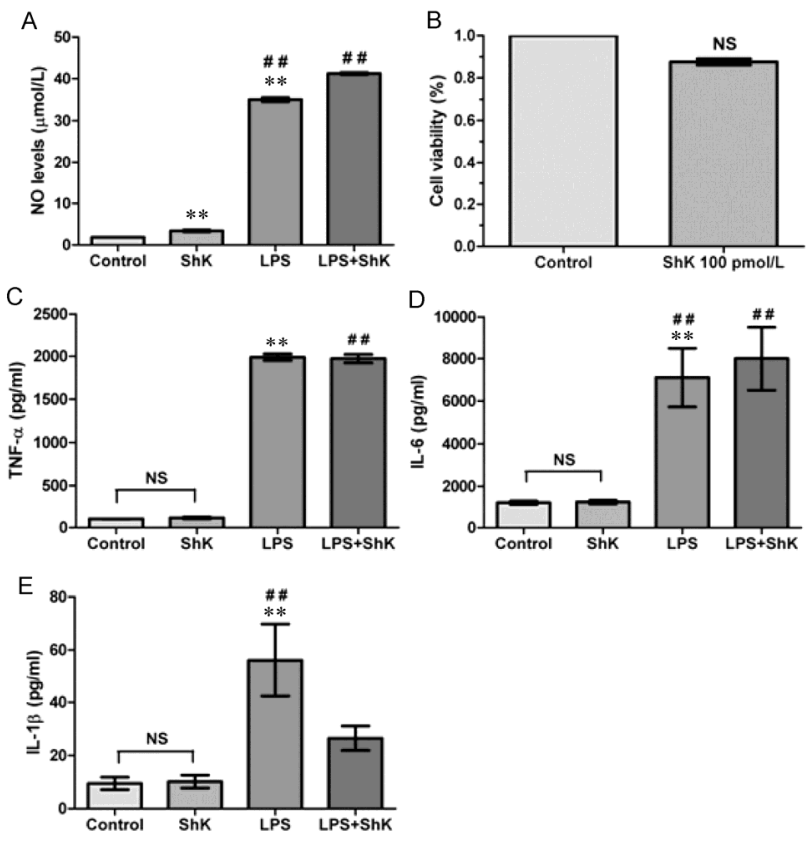

Figure 4 Effects of Kv1.3 blockade on NO production, cell viability, and cytokines secretion in RAW264.7 cells. A, NO assay. B, CCK-8 assay demonstrating cell viability, relative to that of the control cells set to $100 \%$. C-E, ELISA assays indicating the secretions of TNF- $\alpha$, IL-6, and IL-1 $\beta$, respectively. $* *: P<0.01$, vs. Control; \#\#: $P<0.01$, vs. LPS; NS: not significant (vs. Control); $n=3$ for each treatment. Drug concentrations: SHK, 100 pmol L ${ }^{-1}$; LPS, $10 \mu \mathrm{g} \mathrm{mL}^{-1}$.

RAW264.7 cells (Figure 4E).

2.5 Kv1.3 is expressed in RAW264.7 cells and is upregulated by disrupting the caveolin-dependent endocytosis pathway

Western blot revealed that LPS $\left(10 \mu \mathrm{g} \mathrm{mL}^{-1}\right)$, an activator of endocytosis, moderately decreased the protein expression level of Kv1.3 in RAW264.7 cells (Figure 5). Filipin III $\left(5 \mu \mathrm{g} \mathrm{mL}^{-1}\right)$, an inhibitor of caveolin-dependent endocytosis, strongly stimulated the protein expression of Kv1.3 in RAW264.7 cells (Figure 5). Cytochalasin D $\left(10 \mu \mathrm{g} \mathrm{mL}^{-1}\right)$, an inhibitor of actin polymerization, did not affect Kv1.3 expression relative to that in the control RAW264.7 cells (Figure 5). These results suggest that the pathway of caveolin-dependent endocytosis is associated with the protein expression of Kv1.3 in RAW264.7 macrophages.

\section{Discussion}

Kv1.3 channel is involved in the regulations of membrane potential, $\mathrm{Ca}^{2+}$ signaling, immune response [1], and $\mathrm{T}$ cell activation, adhesion, and migration [23]. Lei et al. [21] reported that blocking of Kv1.3 channel by margatoxin could suppress the transition of mononuclear macrophages to foam cells, which suggested Kv1.3 channel is a therapeutic

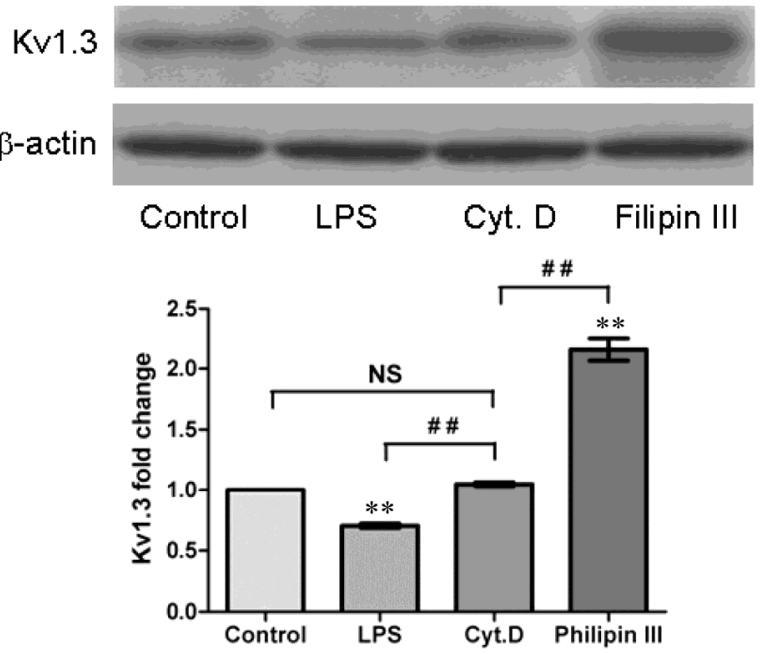

Figure 5 Western blot revealed the protein expression of Kv1.3 in RAW264.7 cells at different treatment conditions. The upper panel represents electrophoresis bands. The lower panel shows the statistical bar graph of Kv1.3 expression levels. **: $P<0.01$, vs. Control; \#\#: $P<0.01$; NS: not significant; $n=3$ for each treatment; Cyt. D: cytochalasin D.

target of atherosclerosis. Despite these findings, the functions of Kv1.3 channel in the macrophages, including that on phagocytosis, remains to be addressed fully. The present study focused on the action of Kv1.3 channel in macrophage phagocytosis. For the first time, we found that, under different treatment conditions, blocking of Kv1.3 channel increases the phagocytic capacity of RAW264.7 macrophages. Our results strongly suggest that Kv1.3 channel acts as a negative regulator of phagocytosis. We further demonstrated that the negative regulation of phagocytosis by $\mathrm{Kv} 1.3$ channel is associated with NO production in activated, but not resting, RAW264.7 cells. However, because Kv1.3 channel agonist is commercially unavailable at present, we could not demonstrate the direct effect of Kv1.3 channel activation on the phagocytosis of macrophages.

We employed two phagocytosis systems to identify the role of Kv1.3 channel in the phagocytic behaviors of RAW264.7 macrophages: the chicken erythrocyte system and the E. coli system. The chicken erythrocyte system [24] is commonly used to measure phagocytosis owing to the unique advantage of visibility of nucleated chicken erythrocytes under a microscope. Through this approach, it is easy to calculate the numbers of macrophages displaying phagocytic behaviors and the numbers of erythrocytes phagocytosed by a macrophage. The E. coli method [25] is also a popular measurement approach of phagocytosis, as bacterial phagocytosis is an innate immune activity in infectious diseases. Using flow cytometry, the E. coli method can accurately and quantitatively estimate the phagocytic activities of macrophages both in vitro and in vivo [26]. In the present study, we used both these methods and found that the results of these two experiments were compatible, suggesting that blocking of Kv1.3 channel by ShK enhances the 
phagocytic activities of RAW264.7 macrophages either on chicken erythrocytes or on FITC-labeled E. coli k-12. The two measurement techniques reciprocally verified the role of Kv1.3 channel in phagocytosis.

The phagocytic activities of macrophages are precisely controlled and regulated under normal conditions, and any dysfunction in the macrophages (overactivity or depressed activity) may lead to or aggravate diseases. Macrophage activation syndrome (MAS) [27] is an example of a disease involving macrophage overactivation. Suppressed phagocytic activities of macrophages are often observed, for example, in the liver Kupffer cells after trauma-hemorrhage [28] and in the alveolar macrophages in chronic obstructive pulmonary disease (unpublished data). Therefore, regulation of the phagocytic capacity of macrophages is an important approach toward treatment of diseases involving macrophage malfunction. We found that blocking of Kv1.3 channel could increase the phagocytic capacity of RAW264.7 macrophages, which suggests that Kv1.3 may be a potential drug target for adjusting macrophage malfunction in certain diseases. On the other hand, direct opening of Kv1.3 channels by selective Kv1.3 channel agonist may potentially exert an opposite effect on the phagocytic capacity of macrophages as compared with that by Kv1.3 channel blockade. However, studies on the development of selective Kv1.3 channel agonist are ongoing. A limitation of the present study includes failure to directly record Kv1.3 currents for evaluating whether the enhanced phagocytosis induced by ShK is caused by a decrease in the Kv1.3 currents.

We further found that blocking of the Kv1.3 channel by ShK treatment did not significantly affect the NO production in resting RAW264.7 cells, although it enhanced the NO production in LPS-stimulated RAW264.7 cells. This phenomenon is consistent with the observation of phagocytosis capacity in resting RAW264.7 cells, which is a weak phagocytic behavior either on chicken erythrocytes or on $E$. coli, while LPS-activated RAW264.7 cells exhibited strong phagocytosis, and ShK yielded greater enhancement on the phagocytic capacity of LPS-activated RAW264.7 cells than on the resting RAW264.7 cells. These results suggest that NO exerts some effects on the Kv1.3 channel activity, considering that LPS induces NO release, which is in turn associated with $\mathrm{Ca}^{2+}$ mobilization and macrophage activation [29]. Moreover, the plasmic $\mathrm{Ca}^{2+}$ level exerts a feedback effect on the Kv channel activities.

Phagocytosis occurs as an inflammatory response to pathogenic factors in several diseases, and cytokine secretion by macrophages is one of the key behaviors of macrophages during inflammation [30]. On the basis of our finding that Kv1.3 modulates the phagocytic behavior of macrophages, we speculate that Kv1.3 may also affect cytokine secretions in macrophages. This hypothesis is supported by our observation that blocking of Kv1.3 channel with ShK treatment decreased the secretion of IL-1 $\beta$ in LPS-stimulated RAW264.7 cells. ShK itself did not significantly affect the secretions of TNF- $\alpha$, IL-6, and IL- $1 \beta$ in resting
RAW264.7 cells that suggests that modulation of certain cytokine secretion by Kv1.3 channel is macrophage activation-dependent and that Kv1.3 channel participates in the inflammatory responses of macrophages. Activation of Kv1.3 channel may potentially exert a suppressing effect on the hyperactive innate immune and inflammatory responses as well as on cytokine storm in certain diseases [31,32].

In addition to the direct channel activation or blockade, the channel protein expression level may also influence the function of the channel. In the present study, LPS significantly decreased the expression of Kv1.3 in RAW264.7 cells, suggesting that macrophage activation exerts a negative feedback effect on the Kv1.3 expression. As a membrane protein, Kv1.3 undergoes recycling across the membrane via endocytosis and recycling, and it is possible that some specific endocytosis pathways are involved in these processes. We previously have identified the internalization pathways by which RAW264.7 macrophages intake $\mathrm{Fe}_{2} \mathrm{O}_{3}$ nanoparticles [33]. In the present study, we found that blocking the caveolin-dependent endocytosis pathway by filipin III could strongly enhance the Kv1.3 expression in RAW264.7 cells, although blocking actin polymerization by cytochalasin D did not affect the Kv1.3 expression. Some investigators have reported that cytochalasin D suppresses the activities of $\mathrm{Cdc} 42$ and Rac [34], which are the two transcription factors involved in the LPS-activation of macrophages. Despite differential results, our observations suggest that certain endocytosis pathway(s), especially the pathway of caveolin-dependent endocytosis, is involved in the regulation of Kv1.3 membrane expression, although the underlying mechanisms of the same need further investigation. Notably, change in the channel expression is not necessarily equivalent to change in channel activation. Therefore, manipulating channel activation status by using a channel opener or blocker cannot be displaced by manipulating the channel expression via gene molecular strategies.

In this study, we used LPS as an activator of macrophages and as a positive control drug. LPS could induce macrophage activation and IL release as well as increase the $\mathrm{Kv} 1.3 / \mathrm{Kv} 1.5$ ratio [35]. LPS-induced activation of macrophages increased the Kv1.3 currents, but decreased the Kir2.1 currents, and TNF- $\alpha$ mimicked LPS effects [36]. These past studies support that LPS activates Kv1.3 channels and that manipulating the Kv1.3 channel activities may exert a regulatory effect on the phagocytic activities of macrophages.

In summary, we found that blocking of Kv1.3 channel by ShK treatment increases the phagocytic capacity and NO production, but decreases IL-1 $\beta$ secretion, in LPSstimulated RAW264.7 macrophages. The expression of Kv1.3 in RAW264.7 macrophages was downregulated by LPS, but it was upregulated by blocking of caveolin-dependent endocytosis with filipin III. The cumulative results suggest that Kv1.3 is a negative regulator of macrophage phagocytosis that may serve as a target for modulating the phagocytic capacity of macrophages. 
This work was supported by the National Key Basic Research Program of China (2011CB93350), and National Natural Science Foundation of China (31171088, 31471126, 81470540, 81300139).

1 Cahalan MD, Chandy KG. The functional network of ion channels in T lymphocytes. Immunol Rev, 2009, 231: 59-87

2 Qiu M, Campbell T, Breit S. A potassium ion channel is involved in cytokine production. Clin Exp Immuonl, 2002, 130: 67-74

3 Villalonga N, David M, Bielanska J, Vicente R, Comes N, Valenzuela $\mathrm{C}$, Felipe A. Immunomodulation of voltage-dependent $\mathrm{K}^{+}$channels in macrophages: molecular and biophysical consequences. J Gen Physiol, 2010, 135: 135-147

4 Wang J, Huang WL, Wang C, Liu RY. Dynamic process of phagocytosis and forms of macrophage cell death induced by ingestion of apoptotic neutrophils. Sci China Life Sci, 2014, 57: 1018-1023

5 Shirai T, Hilhorst M, Harrison DG, Goronzy JJ, Weyand CM. Macrophages in vascular inflammation-from atherosclerosis to vasculitis. Autoimmunity, 2015, 48: 139-151

6 Yan L, Tan XQ, Chen WX, Zhu H, Cao JM, Liu HR. Enhanced vasoconstriction to $\alpha 1$ adrenoceptor autoantibody in spontaneously hypertensive rats. Sci China Life Sci, 2014, 57: 681-689

7 Vicente R, Escalada A, Coma M, Fuster G, Sánchez-Tilló E, López-Iglesias C, Soler C, Solsona C, Celada A, Felipe A. Differential voltage-dependent $\mathrm{K}^{+}$channel responses during proliferation and activation in macrophages. J Biol Chem, 2003, 278: 46307-46320

8 Vicente R, Escalada A, Villalonga N, Texidó L, Roura-Ferrer M, Martín-Satué M, López-Iglesias C, Soler C, Solsona C, Tamkun MM, Felipe A. Association of Kv1.5 and Kv1.3 contributes to the major voltage-dependent $\mathrm{K}^{+}$channel in macrophages. J Biol Chem, 2006, 281: 37675-37685

9 Lam J, Wulff H. The lymphocyte potassium channels Kv1.3 and KCa3.1 as targets for immunosuppression. Drug Dev Res, 2011, 72: 573-584

10 Gao YD, Hanley PJ, Rinné S, Zuzarte M, Daut J. Calcium-activated $\mathrm{K}^{+}$channel $\left(\mathrm{K}_{\mathrm{Ca}} 3.1\right)$ activity during $\mathrm{Ca}^{2+}$ store depletion and store-operated $\mathrm{Ca}^{2+}$ entry in human macrophages. Cell Calcium, 2010, 48: 19-27

11 Tano JY, Lee RH, Vazquez G. Macrophage function in atherosclerosis: potential roles of TRP channels. Channels (Austin), 2012, 6: 141-148

12 Demaurex N, El Chemaly A. Physiological roles of voltage-gated proton channels in leukocytes. J Physiol, 2010, 588: 4659-4665

13 Moreno C, Prieto P, Macías Á, Pimentel-Santillana M, de la Cruz A, Través P G, Boscá L, Valenzuela C. Modulation of voltage-dependent and inward rectifier potassium channels by 15-epi-lipoxin-A4 in activated murine macrophages: implications in innate immunity. J Immunol, 2013, 191: 6136-6146

14 Vicente R, Escalada A, Soler C, Grande M, Celada A, Tamkun MM, Solsona C, Felipe A. Pattern of $\mathrm{Kv} \beta$ subunit expression in macrophages depends upon proliferation and the mode of activation. $\mathrm{J}$ Immunol, 2005, 174: 4736-4744

15 Yang Y, Wang YF, Yang XF, Wang ZH, Lian YT, Yang Y, Li XW, Gao X, Chen J, Shu YW, Cheng LX, Liao YH, Liu K. Specific Kv1.3 blockade modulates key cholesterol-metabolism-associated molecules in human macrophages exposed to ox-LDL. J Lipid Res, 2013, 54: 34-43

16 Leanza L, Zoratti M, Gulbins E, Szabò I. Induction of apoptosis in macrophages via Kv1.3 and Kv1.5 potassium channels. Curr Med Chem, 2012, 19: 5394-5404

17 Villalonga N, David M, Bielanska J. Immunomodulatory effects of diclofenac in leukocytes through the targeting of Kv1.3 voltage-dependent potassium channels. Biochem Pharmacol, 2010, 80: $858-866$
18 Ell Wulff H, Castle NA, Pardo LA. Voltage-gated potassium channels as therapeutic targets. Nat Rev Drug Discov, 2009, 8: 982-1001

19 Pardo LA. Voltage-gated potassium channels in cell proliferation. Physiology, 2004, 19: 285-292

20 Bradding $\mathrm{P}$, Wulff $\mathrm{H}$. The $\mathrm{K}^{+}$channels $\mathrm{K}_{\mathrm{Ca}} 3.1$ and $\mathrm{Kv} 1.3$ as novel targets for asthma therapy. Brit J Pharmacol, 2009, 157: 1330-1339

21 Lei X, Ma A, Xi Y, Zhang W, Yao Y, Du Y. Inhibitory effects of blocking voltage-dependent potassium channel 1.3 on human monocyte-derived macrophage differentiation into foam cells. J Peking Univ (Health Sci), 2006, 38: 257-261

22 Pennington MW, Harunur Rashid M, Tajhya RB, Beeton C, Kuyucak $\mathrm{S}$, Norton RS. A C-terminally amidated analogue of ShK is a potent and selective blocker of the voltage-gated potassium channel Kv1.3. FEBS Lett, 2012, 586: 3996-4001

23 Matheu MP, Beeton C. Imaging of effector memory $\mathrm{T}$ cells during a delayed-type hypersensitivity reaction and suppression by Kv1.3 channel block. Immunity, 2008, 29: 602-614

24 Datta K, Soni JL, Awadhiya RP, Datta IC. Erythrophagocytosis in phenylhydrazine induced acute anaemia in chickens. Res Vet Sci, 1989, 47: 136-137

25 Wang M, Zhao D, Yang Y, Liu J, Wang J, Yin X, Yang L, Zhou X. The cellular prion protein negatively regulates phagocytosis and cytokine expression in murine bone marrow-derived macrophages. PLoS One, 2014, 9: e102785

26 Tartaro K, VanVolkenburg M, Wilkie D, Coskran TM, Kreeger JM, Kawabata TT, Casinghino S. Development of a fluorescence-based in vivo phagocytosis assay to measure mononuclear phagocyte system function in the rat. J Immunotoxicol, 2014, 16: 1-8

27 Zhang M, Behrens EM, Atkinson TP, Shakoory B, Grom AA, Cron RQ. Genetic defects in cytolysis in macrophage activation syndrome. Curr Rheumatol Rep, 2014, 16: 439

28 Hsieh CH, Nickel EA, Chen J, Schwacha MG, Choudhry MA, Bland KI, Chaudry IH. Mechanism of the salutary effects of estrogen on Kupffer cell phagocytic capacity following trauma-hemorrhage: pivotal role of Akt activation. J Immunol, 2009, 182: 4406-4414

29 Zhou X, Yang W, Li J. Ca ${ }^{2+}$ - and protein kinase C-dependent signaling pathway for nuclear factor- $\kappa \mathrm{B}$ activation, inducible nitric-oxide synthase expression, and tumor necrosis factor- $\alpha$ production in lipopolysaccharide-stimulated rat peritoneal macrophages. J Biol Chem, 2006, 281: 31337-31347

30 Turner MD, Nedjai B, Hurst T, Pennington DJ. Cytokines and chemokines: at the crossroads of cell signalling and inflammatory disease. Biochim Biophys Acta, 2014, 1843: 2563-2582

31 Schulert GS, Grom AA. Macrophage activation syndrome and cytokine-directed therapies. Best Pract Res Clin Rheumatol, 2014, 28: 277-292

32 Striz I, Brabcova E, Kolesar L, Sekerkova A. Cytokine networking of innate immunity cells: a potential target of therapy. Clin Sci (Lond), 2014, 126: 593-612

33 Gu J, Xu H, Han Y, Dai W, Hao W, Wang C, Gu N, Xu H, Cao J. The internalization pathway, metabolic fate and biological effect of superparamagnetic iron oxide nanoparticles in the macrophage-like RAW264.7 cell. Sci China Life Sci, 2011, 54: 793-805

34 Kong L, Ge BX. MyD88-independent activation of a novel actin-Cdc42/Rac pathway is required for Toll-like receptor-stimulated phagocytosis. Cell Res, 2008, 18: 745-755

35 Martínez-Mármol R, Villalonga N, Solé L, Vicente R, Tamkun MM, Soler C, Felipe A. Multiple Kv1.5 targeting to membrane surface microdomains. J Cell Physiol, 2008, 217: 667-673

36 Vicente R. Differential voltage-dependent $\mathrm{K}^{+}$channel responses during proliferation and activation in macrophages $\mathrm{J}$ Biol Chem, 2003, 278: 46307-46320

Open Access This article is distributed under the terms of the Creative Commons Attribution License which permits any use, distribution, and reproduction in any medium, provided the original author(s) and source are credited. 